\section{F-Secure: Deutsche besorgt wegen Zugriffe von Unbefugten auf in der Cloud Inhalte}

Das Thema Datenschutz steht aktuell auf der Tagesordnung - aber auch vor dem Fall PRISM waren die Verbraucher besorgt über die Sicherheit und Vertraulichkeit ihrer Daten in der Cloud. Eine weltweit in 15 Ländern durchgeführte und am 27.06.2013 veröffentlichte Umfrage von F-Secure zeigt, dass schon vor den Nachrichten über PRISM sechs von zehn Verbrauchern über die Sicherheit der Speicherung ihrer Inhalte in Social-Networking- und Cloud-Storage-Diensten besorgt waren. 59 Prozent der Verbraucher - in Deutschland 52 Prozent - äußerten sich besorgt darüber, dass jemand anderes auf ihre bei diesen Anbietern gespeicherten Inhalte zugreifen könnte. Dabei sind vor allem jüngere Menschen im Alter von 20 bis 30 Jahren sowie Anwender, die mit verschiedenen Geräten ins Internet gehen und daher in der Regel auch am meisten Cloud-Lösungen beanspruchen, besonders sensibilisiert. Im Rahmen seiner "Digital Lifestyle Survey 2013“ hat F-Secure im April 2013 6.000 Menschen in 15 Ländern befragt.

\section{Sensibilität der Technik-Affinen}

Gerade Anwender, die mit mehreren verschiedenen Geräten digital ins Netz gehen (sogenannte Multi-Screener), waren dabei skeptischer als diejenigen, die meist nur einen Bildschirm nutzten. Gerade diese Gruppe ist auch diejenige, die Cloud-Services am stärksten nutzen. 33 Prozent der Verbraucher haben etwa das Gefühl, dass sie die Kontrolle über ihre Inhalte verlieren, und bei Multi-Screenern waren es noch mehr. Diese Zahl ist am höchsten in Belgien, wo 51 Prozent empfanden, sie verlieren die Kontrolle, und mit 20 Prozent in Großbritannien am niedrigsten. In Deutschland liegt der Wert bei 45 Prozent.

\section{Die beliebtesten Cloud-Speicherplatz-Anbieter}

Laut der Umfrage, sind die fünf großen Anbieter, bei denen Anwender unter anderem ihre Inhalte einmal in der Woche oder täglich hochladen, Facebook (24 Prozent der Befragten), YouTube (14 Prozent), Dropbox (12 Prozent) sowie Apple iCloud und Google Drive mit jeweils 10 Prozent. Ebenfalls 10

Prozent nutzen die Dienste ihres Internet Service Providers.

Weitere Informationen finden sich unter: www.f-secure.com/ content-anywhere<http://bite-de.emailsrvc.net/t/2433/862/601105/2/>

\section{Bundesdruckerei erwirbt Anteile am Kryptografie-Spezialisten cryptovision}

Die Bundesdruckerei GmbH beteiligt sich mit 25,1 Prozent an der cv cryptovision GmbH mit Sitz in Gelsenkirchen. Das Berliner Unternehmen erweitert damit seine Kompetenzen im Bereich hocheffizienter Verschlüsselungstechniken und baut seine Marktposition als Full-Service-Anbieter im Bereich komplexer ID-Sicherheitssysteme aus. Die Beteiligung tritt rückwirkend zum 1. Januar 2013 in Kraft.

cryptovision ist einer der führenden Anbieter für sichere elektronische Identitäten und den Schutz von digitaler Information. Als Spin-off der Universität Essen gegründet, spezialisierte sich das Unternehmen auf moderne kryptografische Verfahren und Public-Key-Infrastrukturen (PKI) für den hoheitlichen und privatwirtschaftlichen Bereich. Im Auftrag des Bundesamts für Sicherheit in der Informationstechnik (BSI) war cryptovision maßgeblich an der Entwicklung des elektronischen Reisepassstandards EAC (Extended Access Control) beteiligt. International gefragt ist auch das seit 2011 am Markt verfügbare Produkt cv act ePasslet suite, eine vollständige Zusammenstellung von Applikationen für hoheitliche elektronische Dokumente. Anwendung findet es beispielsweise bei den elD-Karten von Ruanda und Armenien sowie den elektronischen Reisepässen von Moldawien und Ecuador. Darüber hinaus baut das Unternehmen aktuell die PKI zur Einführung einer eID-Karte in Nigeria auf, mit der digitale Zertifikate ausgestellt, verteilt und geprüft werden können.

Die Lösungen beider Unternehmen ergänzen sich auf optimale Weise, wie gemein-same Projekte bereits gezeigt haben: Die kryptographische Basis-Technologie von cryptovision nutzt die Bundesdruckerei unter anderem für ihre Produkte der Reihe VISOTEC ${ }^{\circledR}$ Border Control. „Aufgrund der guten Zusammenarbeit in den letzten Jahren freuen wir uns, die Bundesdruckerei im Kreise unserer Gesellschafter zu begrü-ßen“, so Markus Hoffmeister, Geschäftsführer (CEO) von cryptovision. „Besonders im Bereich der elD-Spitzentechnologie können so zukünftig weitere Synergien gehoben werden."

\section{Jahre Datenschutz in der Schweiz - wie weiter?}

Am 01.07.2093 - vor 20 Jahren trat das Bundesgesetz über den Datenschutz in Kraft. Grund genug für den Eidgenössischen Datenschutz- und Öffentlichkeitsbeauftragten (EDÖB) Hanspeter Thür, seine Jahrespressekonferenz nicht wie üblich hauptsächlich dem Blick zurück zu widmen, sondern in einer Podiumsdiskussion mit Gästen nach vorne zu schauen: 20 Jahre Datenschutz - wie weiter?

Bereits in der Botschaft aus dem Jahre 1988 an das Parlament sprach der Bundesrat davon, «dass immer größere Mengen von personenbezogenen Daten in immer komplexerer Weise bearbeitet werden», und erblickt darin eine wachsende Gefahr von Persönlichkeitsverletzungen. Zum Schutz der Betroffenen trat am 1. Juli 1993 das Datenschutzgesetz in Kraft. Seit-her hat sich die Realität weiter verändert; rasante Entwicklungen im Bereich Informations- und Kommunikationstechnologien und weltweiter Terrorismus sind nur zwei Herausforderungen für den Schutz der Privatsphäre und die Wahrung des Grundrechts auf informationelle Selbstbestimmung.

Der 20. Tätigkeitsbericht, der den Zeitraum vom April 2012 bis März 2013 abdeckt, wird an diesem Anlass im Berner Käfigturm ebenfalls angesprochen. Der EDÖB legt darin Rechenschaft ab über seine Projekte und Beratungstätigkeiten, greift aktuelle Entwicklungen und relevante Gerichtsentscheide auf und publiziert die Statistiken des Amtsjahres.

\section{Allgemeine Datenschutzfragen}

Wie der EDÖB aufgrund von Bürgeranfragen feststellen musste, überwachen immer mehr Betreiber von Freizeitanlagen sensible Bereiche wie Umkleidekabinen oder Toiletten mit Videokameras. Dieser Trend ist aus Sicht des Datenschutzes höchst bedenklich, wird so doch in die Intimsphäre der betroffenen Personen eingegriffen. Der EDÖB hat dazu Erläuterungen verfasst.

Das in der Schweiz von vielen Skistationen verwendete Zutrittskontrollsystem muss im Bereich der Datensicherheit verbes- 